\title{
Synthesis and Antiproliferative Activity of Novel Limonene Derivatives with a Substituted Thiourea Moiety
}

\author{
Isis M. Figueiredo, ${ }^{a}$ Luciane V. dos Santos, ${ }^{a}$ Willian F. da Costa, ${ }^{a}$ João E. de Carvalho, ${ }^{b}$ \\ Cleuza C. da Silva, ${ }^{a}$ Juliana L. Sacoman, ${ }^{b}$ Luciana K. Kohn ${ }^{b}$ and Maria H. Sarragiotto ${ }^{*, a}$ \\ ${ }^{a}$ Departamento de Química, Universidade Estadual de Maringá, Avenida Colombo 5790, \\ 87020-900 Maringá-PR, Brazil \\ ${ }^{b}$ Centro Pluridisciplinar de Pesquisas Químicas, Biológicas e Agrícolas, Universidade Estadual de Campinas, \\ CP 6171, 13083-970 Campinas-SP, Brazil
}

\begin{abstract}
No presente trabalho descrevemos a síntese e a avaliação da atividade antiproliferativa, frente a linhagens de células tumorais humanas, de derivados do $R$-(+)-limoneno (3-18) contendo uma unidade tiouréia substituída. Os derivados com substituintes arílicos (3-6) exibiram atividade citostática frente a todas linhagens testadas, com inibição de $50 \%$ do crescimento celular $\left(\mathrm{GI}_{50}\right.$ ) em concentrações na faixa de 2,5 a $24 \mu \mathrm{mol} \mathrm{L}-1$. Os compostos 3, 10, 12 e 16 foram os mais ativos, com $\mathrm{GI}_{50}$ na faixa de 0,41 a $3,0 \mu \mathrm{mol} \mathrm{L^{-1 }}$, frente a diferentes linhagens celulares.

A series of $R$-(+)-limonene derivatives bearing a substituted thiourea moiety (3-13) and five S-methyl analogs (14-18) were synthesized and evaluated for their in vitro antiproliferative activity against human cancer cell lines. Compounds bearing aromatic substituents (3-6) exhibit cytotastic activity in the full panel of cell lines tested, with $\mathrm{GI}_{50}$ values in the range of 2.5 to $24 \mu \mathrm{mol} \mathrm{L}{ }^{-1}$. Compounds $3, \mathbf{1 0}, 12$ and 16 were the most active

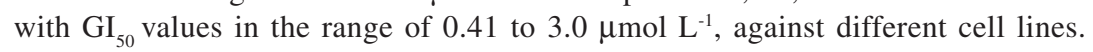

Keywords: limonene derivatives, thioureas, antiproliferative activity

\section{Introduction}

In the last years several approaches have been employed for cancer therapy, and to discover and develop novel therapeutic agents for the treatment of malignancy. In this context, the use of natural products as prototypes has been pointed out as one of the successful approaches to discover novel anticancer drugs.

Monoterpenes are a class of compounds, which occurs naturally in plant, and possess a range of pharmacological properties. Several studies have demonstrated the efficacy of this class of compounds as potential anticancer agents..$^{1-3} \mathrm{D}$-Limonene, a monoterpene found in a variety of foods and essential oils, have been shown to exert chemopreventive and chemotherapeutic activities in a variety of carcinogen-induced animal

*e-mail: mhsarragiotto@uem.br tumor models. ${ }^{4-7}$ Dietary administration of D-limonene causes complete regression of N-nitrosourea (NMU)induced and 7,12-dimethylbenzyl[a]anthracene (DMBA)-induced mammary carcinomas with minimal toxicity. ${ }^{8,9}$

As a result of its chemopreventive and chemotherapeutic potential, limonene is under clinical trials. Phase I and pharmacokinetic study in patients with advanced cancer confirm its low toxicity and support D-limonene as prototype of a novel class of chemotherapeutic drugs. ${ }^{10}$

The potential use of limonene as an anticancer agent led us to focus our attention on the synthesis and antiproliferative activity evaluation of new limonene derivatives, as part of our research program in this area. We present herein the synthesis and the results of the antiproliferative activity evaluation of a series of limonene derivatives (3-13) bearing a substituted thiourea moiety, and of five their S-methyl analogues (14-18). 


\section{Results and Discussion}

\section{Synthesis}

The synthetic route for the preparation of limonene derivatives is presented in Scheme 1. The limonene isothiocyanate (2) was obtained from the reaction of the limonene (1) with $\mathrm{HSCN}$, in chloroform, according to a previously reported procedure. ${ }^{11,12}$ Treatment of $\mathbf{2}$ with different amines (primary, heterocyclic and aromatic) afforded compounds 3-13. For primary and heterocyclic amines the reactions were carried out at $25{ }^{\circ} \mathrm{C}$ for $15 \mathrm{~h}$, by using 2 equivalents of the amine in chloroform as solvent, and the title thioureas were obtained in $67-97 \%$ yield. However, limited yields (20-30\%) were observed for the reaction of 2 with aromatic amines such as aniline, $o$-toluidine, $o$ anisidine and $p$-bromoaniline. In these cases, reactions were performed with a large excess of amine, at $100{ }^{\circ} \mathrm{C}$ for $24 \mathrm{~h}$. The products (3-13) were characterized by analysis of their spectroscopic data. The presence of the $\mathrm{C}=\mathrm{S}$ group was evidenced by the IR absorption bands at 1523-1566 and 1250-1410 $\mathrm{cm}^{-1}$, together with the signal at $\delta 177.3-181.8$ in the ${ }^{13} \mathrm{C}$ NMR spectra. The monoterpene moiety was characterized by the signals at $\delta_{\mathrm{H}} / \delta_{\mathrm{C}} 5.30-5.40(1 \mathrm{H}$, brs, $\mathrm{H}-2) / 119.7-120.5$ (C-2), 1.30-1.49 (3H, s, H-8)/24.1-24.9 (C-8), 1.34-1.50 $(3 \mathrm{H}, \mathrm{s}, \mathrm{H}-9) / 24.2-25.4(\mathrm{C}-9)$ and $1.61-1.73(3 \mathrm{H}, \mathrm{s}, \mathrm{H}-$ 10)/23.0-23.5 (C-10) in the ${ }^{1} \mathrm{H} /{ }^{13} \mathrm{C}$ NMR spectra. The NMR data were also consistent for the substituents attached to the nitrogen atom $\mathrm{N}-1$, as showed in the Experimental Section. The EI-mass spectra showed peaks at $m / z 58$ and at $m / z\left(\mathbf{M}^{+*}-135\right)$ as main fragments.

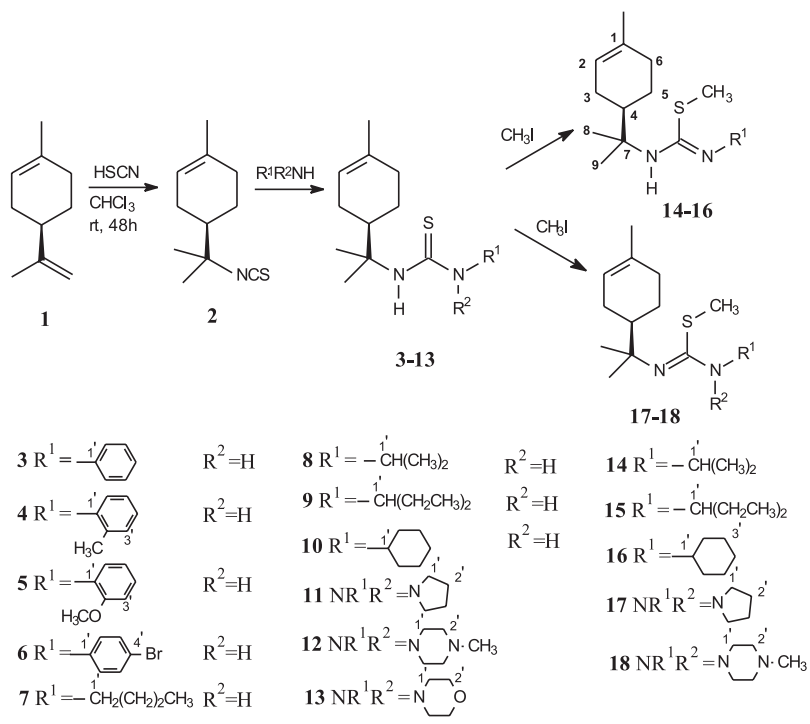

Scheme 1.
The proposed mechanisms for the fragmentation are shown in Figure 1.

The S-methylated derivatives (14-18) were prepared from the reaction of the corresponding thioureas with methyl iodide at $0{ }^{\circ} \mathrm{C}$, in chloroform, for $24 \mathrm{~h}$ in quantitative yields. The ${ }^{1} \mathrm{H}$ NMR spectra of the Smethylthioureas showed signal at $\delta 2.78-2.85$ corresponding to the $\mathrm{SCH}_{3}$ group. The formation of Smethythiourea was also evidenced by the presence of the signals at $\delta 16.9-18.5\left(\mathrm{~S}^{-\mathrm{CH}_{3}}\right)$ and $\delta 143.7-169.2(\mathrm{C}=\mathrm{N})$ in the ${ }^{13} \mathrm{C}$ NMR spectra, besides of the absorption at 1600 $1690 \mathrm{~cm}^{-1}(\mathrm{C}=\mathrm{N})$, in the IR spectra.

\section{Antiproliferative activity}

The results of the antiproliferative assays are showed in Tables 1 and 2. The response parameter $\mathrm{GI}_{50}$ (Table 1) refers to the drug concentration that produce a $50 \%$ reduction of cellular growth when compared to untreated control cells. Table 2 includes data for the compounds that reached TGI and $\mathrm{LC}_{50}$ values. The TGI and $\mathrm{LC}_{50}$ parameters refer, respectively, to the drug concentration for total growth inhibition, and that for killing 50\% of the cells.

As shown in Table 1, the compounds bearing aromatic substituents (3-6) exhibit cytotastic activity against all cancer cell lines tested, with $\mathrm{GI}_{50}$ values in the range of 2.5 to $24 \mu \mathrm{mol} \mathrm{L}^{-1}$. Analysis of TGI and $\mathrm{LC}_{50}$ data presented in Table 2 show that compounds 3-5 had higher potency for total growth inhibition, and for killing 50\% of the cells. From this series, compound $\mathbf{3}$ was the most active, with $\mathrm{GI}_{50}$ and TGI values of $2.5 \mu \mathrm{mol} \mathrm{L}^{-1}$ and $22.5 \mu \mathrm{mol} \mathrm{L}^{-1}$, respectively, against breast resistant $\mathrm{NCI} /$ ADR cancer cell line. On the other hand, compounds 718 of the aliphatic series exhibit different profiles and dependence on the nature of the substituent on the nitrogen atom. Compared to aromatic series, the specificity was increased with the most of the compounds

a)

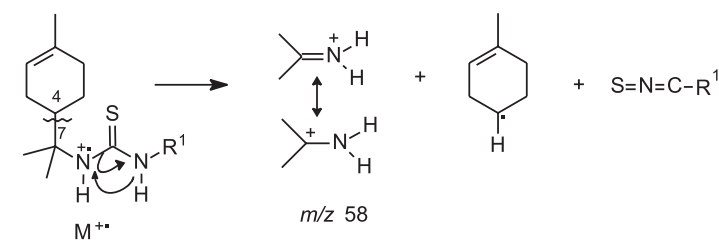

b)

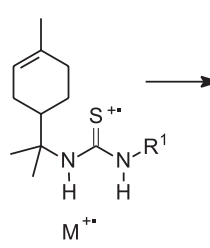

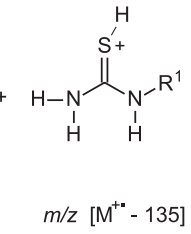

Figure 1. Proposed mechanism for formation of the fragments a) $\mathrm{m} / \mathrm{z} .58$ and b) $m / z\left(\mathbf{M}^{+}-135\right)$. 
Table 1. $\mathrm{GI}_{50}$ values (in $\mu \mathrm{mol} \mathrm{L}{ }^{-1}$ concentrations) of the limonene derivatives (3-18)

\begin{tabular}{|c|c|c|c|c|c|c|c|c|c|c|c|}
\hline \multirow[b]{2}{*}{ Compounds } & \multirow[b]{2}{*}{$\mathrm{R}^{1}$} & \multirow[b]{2}{*}{$\mathrm{R}^{2}$} & \multicolumn{9}{|c|}{ Cancer cell lines } \\
\hline & & & $\begin{array}{l}\text { Melanoma } \\
\text { UACC-62 }\end{array}$ & $\begin{array}{l}\text { Breast } \\
\text { MCF7 }\end{array}$ & $\begin{array}{c}\text { Lung } \\
\text { NCI-460 }\end{array}$ & $\begin{array}{l}\text { Leukemia } \\
\text { K-562 }\end{array}$ & $\begin{array}{l}\text { Ovarian } \\
\text { OVCAR }\end{array}$ & $\begin{array}{c}\text { Prostate } \\
\text { PCO-3 }\end{array}$ & $\begin{array}{l}\text { Colon } \\
\text { HT29 }\end{array}$ & $\begin{array}{l}\text { Renal } \\
786-0\end{array}$ & $\begin{array}{c}\text { Breast resistant } \\
\text { NCI/ADR }\end{array}$ \\
\hline \multicolumn{12}{|c|}{ Aromatic } \\
\hline 3 & $\mathrm{Ph}$ & $\mathrm{H}$ & 13.9 & 18.4 & 17.3 & $\mathrm{nt}$ & 16.6 & 15.6 & 24.0 & 23.2 & 2.5 \\
\hline 4 & 2-Me-Ph & $\mathrm{H}$ & 12.0 & 21.0 & 18.3 & 18.7 & 17.2 & 19.8 & 9.3 & 16.1 & 12.4 \\
\hline 5 & 2-OMe-Ph & $\mathrm{H}$ & 11.8 & 13.6 & 15.0 & 10.5 & 9.5 & 11.0 & 18.2 & 12.7 & 8.0 \\
\hline 6 & $\begin{array}{l}\text { 4-Br-Ph } \\
\text { Alipha }\end{array}$ & $\mathrm{H}$ & $\mathrm{nt}$ & 21.5 & 19.9 & 20.1 & 16.7 & 16.5 & 14.6 & 20.9 & 19.0 \\
\hline 7 & n-Butyl & $\mathrm{H}$ & $>100$ & $>100$ & $>100$ & 39.5 & 98.1 & $>100$ & 60.6 & 92.4 & 54.1 \\
\hline 8 & i-Propyl & $\mathrm{H}$ & 31.8 & 70.7 & 29.5 & 18.9 & 28.3 & $>100$ & 30.7 & 32.6 & 13.8 \\
\hline 9 & i-Pentyl & $\mathrm{H}$ & 97.7 & 46.5 & 54.3 & 6.6 & 56.2 & 62.6 & 17.5 & 92.1 & 32.7 \\
\hline 10 & Cyclohexyl & $\mathrm{H}$ & nt & 37.3 & 33.9 & 16.3 & 3.0 & 12.5 & $\mathrm{nt}$ & 26.5 & 6.1 \\
\hline 11 & \multicolumn{2}{|c|}{ Pyrrolidyl } & 53.3 & 26.1 & 34.5 & 23.6 & 44.3 & 43.0 & 36.8 & 59.6 & 28.5 \\
\hline 12 & \multicolumn{2}{|c|}{ 1-N-Methylpiperazyl } & $>100$ & $>100$ & $>100$ & 3.0 & $>100$ & $>100$ & $>100$ & $>100$ & 88.0 \\
\hline 13 & \multicolumn{2}{|c|}{ Morpholyl } & $>100$ & $>100$ & $>100$ & $>100$ & $>100$ & $>100$ & $>100$ & $>100$ & $>100$ \\
\hline \multicolumn{12}{|c|}{ S-Methylthioureas } \\
\hline 14 & \multicolumn{2}{|l|}{ i-Propyl } & 83.8 & $>100$ & $>100$ & 25.4 & $>100$ & $>100$ & 81.8 & $>100$ & 85.3 \\
\hline 15 & \multicolumn{2}{|l|}{ i-Pentyl } & 28.9 & 16.0 & 17.6 & 88.7 & 91.3 & 16.2 & 16.7 & 14.8 & 10.3 \\
\hline 16 & \multicolumn{2}{|l|}{ Cyclohexyl } & 60.7 & 60.7 & 58.9 & 0.41 & 39.1 & 62.5 & 16.7 & 75.6 & 75.6 \\
\hline 17 & \multicolumn{2}{|c|}{ Pyrrolidyl } & 90.0 & $>100$ & $>100$ & $>100$ & 60.9 & $>100$ & $>100$ & $>100$ & 91.3 \\
\hline 18 & \multicolumn{2}{|c|}{ 1-N-Methylpiperazyl } & $>100$ & $>100$ & $>100$ & 76.7 & $>100$ & $>100$ & $>100$ & $>100$ & $>100$ \\
\hline \multicolumn{3}{|l|}{ Doxorubicin } & 2.36 & 8.84 & 1.21 & 3.04 & 9.67 & 7.88 & 2.73 & 1.76 & $>100$ \\
\hline
\end{tabular}

nt $=$ not tested.

Table 2. TGI values and $\mathrm{LC}_{50}$ (values in parenthesis), in $\mu \mathrm{mol} \mathrm{L}^{-1}$ concentrations

\begin{tabular}{|c|c|c|c|c|c|c|c|c|c|c|c|}
\hline \multirow[b]{2}{*}{ Compounds } & \multirow[b]{2}{*}{$\mathrm{R}^{1}$} & \multirow[b]{2}{*}{$\mathrm{R}^{2}$} & \multicolumn{9}{|c|}{ Cancer cell lines } \\
\hline & & & $\begin{array}{r}\text { Melanoma } \\
\text { UACC-62 }\end{array}$ & $\begin{array}{l}\text { Breast } \\
\text { MCF7 }\end{array}$ & $\begin{array}{c}\text { Lung } \\
\text { NCI-460 }\end{array}$ & $\begin{array}{c}\text { Leukemia } \\
\text { K-562 }\end{array}$ & $\begin{array}{r}\text { Ovarian } \\
\text { OVCAR }\end{array}$ & $\begin{array}{c}\text { Prostate } \\
\text { PCO-3 }\end{array}$ & $\begin{array}{l}\text { Colon } \\
\text { HT29 }\end{array}$ & $\begin{array}{l}\text { Renal } \\
786-0\end{array}$ & $\begin{array}{c}\text { Breast resistant } \\
\text { NCI/ADR }\end{array}$ \\
\hline \multicolumn{12}{|l|}{ Thioureas } \\
\hline 3 & $\mathrm{Ph}$ & $\mathrm{H}$ & 34.6 & 55.5 & 86.6 & $>100$ & $>100$ & 58.9 & $>100$ & 86.6 & 22.5 (76.6) \\
\hline 4 & 2-Me-Ph & $\mathrm{H}$ & $29.7(80.9)$ & 86.9 & 47.2 & $>100$ & 49.5 & $>100$ & $>100$ & $29.8(65.0)$ & ) $30.0(65.0)$ \\
\hline 5 & 2-OMe-Ph & $\mathrm{H}$ & 39.2 & 64.6 & 39.5 & $>100$ & 32.0 & 58.0 & $>100$ & $26.8(57.7)$ & 26.9 \\
\hline 6 & 4-Br-Ph & $\mathrm{H}$ & $>100$ & $>100$ & 71.0 & $>100$ & 72.0 & $>100$ & 95.0 & 71.0 & 71.0 \\
\hline 9 & i-Pentyl & $\mathrm{H}$ & $>100$ & $>100$ & $>100$ & 95.0 & $>100$ & $>100$ & $>100$ & $>100$ & $>100$ \\
\hline \multicolumn{12}{|c|}{ S-Methylthioureas } \\
\hline 15 & i-Pentyl & & $>100$ & 68.3 & 87.8 & $>100$ & $>100$ & $>100$ & 87.8 & 58.0 & 70.6 \\
\hline 16 & Cyclohexyl & & $>100$ & $>100$ & $>100$ & 82.5 & $>100$ & $>100$ & 93.0 & $>100$ & $>100$ \\
\hline \multicolumn{2}{|l|}{ Doxorubicin } & & 64.0 & & 6.86 & $>100$ & $>100$ & 92.1 & & $>100$ & \\
\hline
\end{tabular}

of the aliphatic series exhibiting smaller $\mathrm{GI}_{50}$ values for leukemic K-562 cell line. From the thiourea aliphatic series, compounds $\mathbf{9}$ and $\mathbf{1 2}$ showed potent activity and particular selectivity against leukemic K-562 cell line

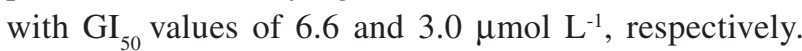
The replacement of the 1-N-methyl group of the compound $\mathbf{1 2}$ for an oxygen results in the inactive compound 13. The thiourea 10 exhibits potent antiproliferative activity against ovarian OVCAR and breast resistant NCI/ADR with $\mathrm{GI}_{50} 3.0$ and $6.1 \mu \mathrm{mol}$ $\mathrm{L}^{-1}$, respectively. S-methylation of thioureas bearing aliphatic substituents results in changes in the antiproliferative activity profiles. Conversion of the thiourea $\mathbf{1 0}$ to the S-methylthiourea analogue 16, led to the most potent compound, with $\mathrm{GI}_{50}$ value of $0.41 \mu \mathrm{mol}$ $\mathrm{L}^{-1}$ and high selectivity against leukemia $\mathrm{K}-562$ cell lines.

\section{Conclusions}

In this paper we report the synthesis and cytotoxic evaluation of a series of new limonene derivatives containing a substituted thiourea moiety. The results show the potentiality of some compounds, particularly $\mathbf{3}, \mathbf{1 0}$, 12 and 16, as inhibitors of tumor cells proliferation. Some compounds bearing aromatic substituents were also able to kill $50 \%$ of the breast resistant NIC/ADR (compounds 
3 and 4), melanoma UACC-62 (compound 4), and renal 786-0 (compounds $\mathbf{4}$ and $\mathbf{5}$ ) cancer cell lines.

\section{Experimental}

IR spectra were recorded on $\mathrm{KBr}$ pellets in a Bomem model MB-100 spectrophotometer. Mass spectra were measured on Shimadzu GC/MS, QP 2000A, at $70 \mathrm{eV} .{ }^{1} \mathrm{H}$ and ${ }^{13} \mathrm{C}$ NMR spectra were recorded on a Varian Mercury Plus $300 \mathrm{MHz}$ in $\mathrm{CDCl}_{3}$ and TMS as internal reference. Column chromatography was performed on silica gel Merck 230-400 mesh ASTM.

\section{General procedure for thioureas $\mathbf{3}-\mathbf{1 3}$}

To a solution of limonene isothiocyanate 2 (1 mmol) in $\mathrm{CHCl}_{3}(10 \mathrm{~mL})$ was added drop-wise the amine $(2$ mmol). ${ }^{11,12}$ The solution was kept at room temperature for 15 hours and then the solvent was removed under reduced pressure. For aromatic amines, reactions were performed with a large excess of amine, without solvent, at $100{ }^{\circ} \mathrm{C}$ for $24 \mathrm{~h}$. The residue was purified by column chromatography using hexane and a mixture of hexane-ethyl acetate in increasing polarity as solvent.

$N$-[1-(4R)-(4-isopropyl-1-methylcyclohexenyl)]-N'-[2(phenyl)]thiourea (3). Yield: $30 \%$; IR $v_{\max } / \mathrm{cm}^{-1}: 3387(\mathrm{~N}-$ H), 3156 (C-H arom), 1590 (C=C arom), 1540 and 1298 $(\mathrm{C}=\mathrm{S})$; EI-MS $m / z$ (rel. int.): $288\left(\mathrm{M}^{+*}, 5\right), 153\left(100, \mathrm{M}^{+*}\right.$ $\left.135,\left[\mathrm{H}_{2} \mathrm{NCSNHC}_{6} \mathrm{H}_{5}\right]^{+}\right) ;{ }^{1} \mathrm{H} \mathrm{NMR}\left(300 \mathrm{MHz}, \mathrm{CDCl}_{3}\right)$ : $\delta 1.18$ and $1.62(1 \mathrm{H}$ each, $\mathrm{m}, \mathrm{H}-5), 1.40(3 \mathrm{H}, \mathrm{s}, \mathrm{H}-8)$, $1.45(3 \mathrm{H}, \mathrm{s}, \mathrm{H}-9), 1.61(3 \mathrm{H}, \mathrm{s}, \mathrm{H}-10), 1.84$ and $1.90(4 \mathrm{H}$, m, H-3 and H-6), $2.59(1 \mathrm{H}, \mathrm{m}, \mathrm{H}-4), 5.36(1 \mathrm{H}$, brs, H2), $7.20\left(2 \mathrm{H}, \mathrm{d}, J 7.5 \mathrm{~Hz}, \mathrm{H}-2\right.$ ' and $\left.\mathrm{H}-6{ }^{\prime}\right), 7.29(1 \mathrm{H}, \mathrm{t}, J$ $7.5 \mathrm{~Hz}, \mathrm{H}-4$ '), 7.43 (2H, t, J 7.5 Hz, H-3' and $\mathrm{H}-5$ '); ${ }^{13} \mathrm{C}$ NMR (75.5 MHz, $\left.\mathrm{CDCl}_{3}\right): \delta 23.5(\mathrm{C}-10), 24.2(\mathrm{C}-9)$, 24.3 (C-5), 24.5 (C-8), 26.7 (C-3), 31.2 (C-6), 41.1 (C4), 59.5 (C-7), 120.5 (C-2), 125.2 (C-2' and C-6'), 127.1 (C-4'), 130.2 (C-3' and C-5'), 134.3 (C-1), 136.8 (C1'), $179.5(\mathrm{C}=\mathrm{S})$.

$N-[1-(4 R)-(4-i s o p r o p y l-1-m e t h y l c y c l o h e x e n y l)]-N$ '-[2-(2methylphenyl)]thiourea (4). Yield: $30 \%$. IR $v_{\max } / \mathrm{cm}^{-1}: 3367$ (N-H), 3173 (C-H arom), $1643(\mathrm{C}=\mathrm{C}$ arom), $1621(\mathrm{C}=\mathrm{C})$, 1533 and $1251(\mathrm{C}=\mathrm{S})$; EI-MS $\mathrm{m} / z$ (rel. int.): $302\left(\mathrm{M}^{+*}\right.$, 10), $167\left(100, \mathrm{M}^{+\bullet}-135,\left[\mathrm{H}_{2} \mathrm{NCSNH}-2 \text {-methylphenyl }\right]^{+}\right)$, 91 (45), 58 (70), 41 (46); ${ }^{1} \mathrm{H}$ NMR (300 MHz, $\mathrm{CDCl}_{3}$ ): $\delta$ 1.39 (3H, s, H-8), 1.45 (3H, s, H-9), 1.61 (3H, s, H-10), $1.75(2 \mathrm{H}, \mathrm{m}, \mathrm{H}-5), 1.93$ (4H, m, H-3 and $\mathrm{H}-6), 2.29(3 \mathrm{H}$, s, $\left.\mathrm{CH}_{3}-\mathrm{Ar}\right), 2.48(1 \mathrm{H}, \mathrm{m}, \mathrm{H}-4), 5.30$ (1H, brs, H-2), 7.20 (1H, m, H-6'), 7.25 (1H, m, H-5'), 7.28 (1H, m, H-4'), $7.31\left(1 \mathrm{H}, \mathrm{m}, \mathrm{H}-3\right.$ '); ${ }^{13} \mathrm{C} \mathrm{NMR}\left(75.5 \mathrm{MHz}, \mathrm{CDCl}_{3}\right): \delta 18.1$ ( $\left.\underline{\mathrm{CH}}_{3}-\mathrm{Ar}\right), 23.5$ (C-10), 24.1 (C-8), 24.2 (C-5), 24.4 (C9), 26.6 (C-3), 31.2 (C-6), 41.4 (C-4), 59.2 (C-7), 120.5 (C-2), 127.7 (C-6'), 127.8 (C-5'), 127.9 (C-2'), 128.7 (C4'), 131.9 (C-3'), 134.3 (C-1), 136.0 (C-1'), 179.9 (C=S).

$N$-[1-(4R)-(4-isopropyl-1-methylcyclohexenyl)]-N'-[2(2-methoxyphenyl)]thiourea (5). Yield: $31 \%$; IR $v_{\max } / \mathrm{cm}^{-1}$ : $3370(\mathrm{~N}-\mathrm{H}), 3048$ (C-H arom), 1599 and $1535(\mathrm{C}=\mathrm{C}), 1460$ and $1250(\mathrm{C}=\mathrm{S})$; EI-MS $\mathrm{m} / z$ (rel. int.): $318\left(\mathrm{M}^{+*}, 7\right), 183$ $\left(27, \mathrm{M}^{+*}-135,\left[\mathrm{H}_{2} \mathrm{NCSNH}-2-m e t h o x y p h e n y l\right]^{+}\right) ; 123$ (93, $\left.\left[\mathrm{H}_{2} \mathrm{~N}-2 \text {-methoxyphenyl }\right]^{+}\right), 93$ (27), 58 (100), $41(43) ;{ }^{1} \mathrm{H}$ NMR (300 MHz, $\left.\mathrm{CDCl}_{3}\right): \delta 1.42(3 \mathrm{H}, \mathrm{s}, \mathrm{H}-8), 1.48(3 \mathrm{H}, \mathrm{s}$, H-9), 1.63 (3H, s, H-10), 1.77 (2H, m, H-5), 1.90 (4H, m, $\mathrm{H}-3$ and $\mathrm{H}-6), 2.00$ (1H, m, H-4), 3.84 (3H, s, $\left.\mathrm{OC}_{3}\right), 5.36$ $(1 \mathrm{H}$, brs, H-2), $6.92(1 \mathrm{H}, \mathrm{m}, \mathrm{H}-3$ '), $6.94(1 \mathrm{H}, \mathrm{d}, J 7.8 \mathrm{~Hz}$, H-6'), $6.98(1 \mathrm{H}, \mathrm{t}, J 7.8 \mathrm{~Hz}, \mathrm{H}-5$ '), $7.20(1 \mathrm{H}, \mathrm{t}, J 7.8 \mathrm{~Hz}$, $\mathrm{H} 4$ '); ${ }^{13} \mathrm{C}$ NMR $\left(75.5 \mathrm{MHz}, \mathrm{CDCl}_{3}\right): \delta 23.4(\mathrm{C}-10), 24.3$ (C-5), 24.6 (C-8), 24.6 (C-9), 26.7 (C-3), 31.2 (C-6), 41.7 $(\mathrm{C}-4), 55.8\left(\mathrm{OCH}_{3}\right), 59.3(\mathrm{C}-7), 110.3\left(\mathrm{C}^{\prime} \mathbf{4}^{\prime}\right), 120.5(\mathrm{C}-2)$, 121.0 (C- 5' and C-6'), 124.5 (C-3'), 134.1 (C-1'), 134.2 (C-1), $154.6\left(\mathrm{C}-2^{\prime}\right), 179.3(\mathrm{C}=\mathrm{S})$.

$N$-[1-(4R)-(4-isopropyl-1-methylcyclohexenyl)]-N'-[2-(4bromophenyl)]thiourea (6). Yield: $20 \%$; IR $v_{\max } / \mathrm{cm}^{-1}: 3281$ $(\mathrm{N}-\mathrm{H}), 3103(\mathrm{C}-\mathrm{H}), 1578$ and $1530(\mathrm{C}=\mathrm{C}$, arom.), 1488 and $1364(\mathrm{C}=\mathrm{S})$; EI-MS $\mathrm{m} / z$ (rel. int.): $366\left(\mathrm{M}^{+*}, 7\right), 215$ (23), 58 (100); ${ }^{1} \mathrm{H}$ NMR (300 MHz, $\mathrm{CDCl}_{3}$ ): $\delta 1.24$ and 1.68 (1H each, m, H-5'), 1.42 (3H, s, H-8), 1.48 (3H, s, H-9), 1.63 (3H, s, H-10), 1.68 and $1.97(1 \mathrm{H}$ each, m, H3), $1.96(2 \mathrm{H}, \mathrm{m}, \mathrm{H}-6), 2.59(1 \mathrm{H}, \mathrm{m}, \mathrm{H}-4), 5.36(1 \mathrm{H}, \mathrm{brs}$, H-2), 7.09 (2H, d, $J 8.7 \mathrm{~Hz}, \mathrm{H}-2^{\prime}$ and $\left.\mathrm{H}-6^{\prime}\right), 7.54(2 \mathrm{H}, \mathrm{d}$, $J$ 8.7 Hz, H-3' and $\mathrm{H}-5$ '); ${ }^{13} \mathrm{C} \mathrm{NMR}\left(75.5 \mathrm{MHz}, \mathrm{CDCl}_{3}\right)$ : $\delta 23.5$ (C-10), 24.4 (C-5), 24.6 (C-8), 24.6 (C-9), 26.7 (C-3), 31.2 (C-6), 40.9 (C-4), 59.7 (C-7), 120.4 (C-2), 120.6 (C-4'), 126.8 (C2' and C-6'), 133.4 (C3' and C-5'), 134.4 (C-1), 136.0 (C-1'), $179.4(\mathrm{C}=\mathrm{S})$.

$N$-[1-(4R)-(4-isopropyl-1-methylcyclohexenyl)]-N'-[2(butyl)]thiourea (7). Yield: 97\%; IR $v_{\max } / \mathrm{cm}^{-1}: 3269$ (NH), 1543 and 1342 (C=S); EI-MS $m / z$ (rel. int.): $268\left(\mathrm{M}^{+*}\right.$, 10), $133\left(100, \mathrm{M}^{+*}-135,\left[\mathrm{H}_{2} \mathrm{NCSNH}-b u t y 1\right]^{+}\right), 58$ (68), 41 (60); ${ }^{1} \mathrm{H}$ NMR (300 MHz, $\mathrm{CDCl}_{3}$ ): $\delta 0.95(3 \mathrm{H}, \mathrm{t}, J 7.2 \mathrm{~Hz}$, H-4'); 1.35 (3H, s, H-8), 1.39 (3H, s, H-9), 1.41 (2H, m, H-3'), 1.60 (2H, m, H-6), 1.64 (3H, s, H-10), 1.82 (2H, m, H-5), 1,99 (3H, m, H-3 and H-4), 2.01 (2H, m, H-2'), $3.54\left(2 \mathrm{H}, \mathrm{q}, J 6.0 \mathrm{~Hz}, \mathrm{H}-1\right.$ ') $5.40(1 \mathrm{H}$, brs, $\mathrm{H}-2) ;{ }^{13} \mathrm{C}$ NMR (75.5 MHz, $\mathrm{CDCl}_{3}$ ): $\delta 13.7$ (C-4'), 20.1 (C-3'), 23.2 (C-10), 24.2 (C-5), 24.8 (C-8), 25.0 (C-9), 26.5 (C-3), 30.9 (C-2'), 31.2 (C-6), 58.3 (C-7), 42.2 (C-4), 45.1 (C1'), 120.0 (C-2), 133.9 (C-1), 180.8 (C=S). 
$N$-[1-(4R)-(4-isopropyl-1-methylcyclohexenyl)]-N'-[2(isopropyl)]thiourea (8). Yield: 84\%; IR $v_{\max } / \mathrm{cm}^{-1}: 3272$ (N-H), 1538 and 1325 (C=S); EI-MS m/z (rel. int.): 254 $\left(\mathrm{M}^{+\bullet}, 7\right), 119\left(100, \mathrm{M}^{+\bullet}-135,\left[\mathrm{H}_{2} \mathrm{NCSNH}-\text { isopropyl] }\right]^{+}\right)$, 58 (65), 41 (60); ${ }^{1} \mathrm{H}$ NMR (300 $\mathrm{MHz}, \mathrm{CDCl}_{3}$ ): $\delta 1.23$ $\left(6 \mathrm{H}, \mathrm{d}, J 6.0 \mathrm{~Hz}, \mathrm{NHCH}\left(\mathrm{CH}_{3}\right)_{2}\right), 1.35(3 \mathrm{H}, \mathrm{s}, \mathrm{H}-8), 1.40$ (3H, s, H-9), 1.65 (3H, s, H-10), 1.78 (2H, m, H-5), 1.99 (5H, m, H-3, H-4 and H-6), 4.40 (1H, m, H-1'), 5.40 (1H, brs, H-2); $\left.{ }^{13} \mathrm{C} \mathrm{NMR} \mathrm{(75.5} \mathrm{MHz} \mathrm{CDCl}_{3}\right)$ : $\delta 22.6$ $\left(\mathrm{NHCH}\left(\mathrm{CH}_{3}\right)_{2}\right), 23.2$ (C-10), 24.2 (C-5), 24.9 (C-8), 25.2 (C-9), 26.6 (C-3), 30.9 (C-6), 42.5 (C-4), 47.2 (C-1'), 58.3 (C-7), 120.0 (C-2), 134.0 (C-1), 179.8 (C=S).

$N$-[1-(4R)-(4-isopropyl-1-methylcyclohexenyl)]-N'-[2(isopentyl)]thiourea (9). Yield: $80 \%$; IR $v_{\max } / \mathrm{cm}^{-1}: 3258$ $(\mathrm{N}-\mathrm{H}), 1643(\mathrm{C}=\mathrm{C}), 1537$ and $1322(\mathrm{C}=\mathrm{S})$; (KBr); EI-MS $m / z$ (rel. int.): $282\left(\mathbf{M}^{+\cdot \bullet}, 10\right), 147\left(100, \mathbf{M}^{+\bullet}-135\right.$, $\left.\left[\mathrm{H}_{2} \mathrm{NCSNH} \text {-isopentyl] }\right]^{+}\right), 58$ (70); $41(65) ;{ }^{1} \mathrm{H}$ NMR (300 $\left.\mathrm{MHz}, \mathrm{CDCl}_{3}\right): \delta 0.93\left(6 \mathrm{H}, \mathrm{t}, J 7.5 \mathrm{~Hz}, \mathrm{NHCH}\left(\mathrm{CH}_{2} \mathrm{CH}_{3}\right)_{2}\right)$, 1.30 and 1.83 (1H each, m, H-5), 1.34 (3H, s, H-8), 1.38 $(3 \mathrm{H}, \mathrm{s}, \mathrm{H}-9), 1.51\left(4 \mathrm{H}, \mathrm{m}, \mathrm{NHCH}\left(\mathrm{CH}_{2} \mathrm{CH}_{3}\right)_{2}\right), 1.61(2 \mathrm{H}$, m, H-3), 1.65 (3H, s, H-10), 1.99 (3H, m, H-4 and H-6), 4.23 (1H, m, H-1'), 5.36 (1H, brs, H-2); ${ }^{13} \mathrm{C}$ NMR $(75.5$ $\left.\mathrm{MHz}, \mathrm{CDCl}_{3}\right): \delta 10.0\left(\mathrm{NHCH}\left(\mathrm{CH}_{2} \underline{\mathrm{CH}}_{3}\right)_{2}\right), 23.3(\mathrm{C}-10)$, 24.3 (C-5), 25.1 (C-8), 25.4 (C-9), 26.6 (C-3), 27.0 $\left(\mathrm{NHCH}\left(\mathrm{CH}_{2} \mathrm{CH}_{3}\right)_{2}\right), 31.0(\mathrm{C}-6), 42.8(\mathrm{C}-4), 58.2(\mathrm{C}-7)$, 58.3 (C-1'), 119.7 (C-2), 134.1 (C-1), 180.7 (C=S).

$N$-[1-(4R)-(4-isopropyl-1-methylcyclohexenyl)]-N'-[2(cyclohexyl)]thiourea (10). Yield: 80\%; IR $v_{\max } / \mathrm{cm}^{-1}: 3258$ (N-H); 1540 and $1338(\mathrm{C}=\mathrm{S})$; EI-MS m/z (rel. int.): 294 $\left(\mathrm{M}^{+\bullet}, 10\right), 58$ (100), 41 (60); ${ }^{1} \mathrm{H}$ NMR (300 MHz, $\left.\mathrm{CDCl}_{3}\right)$ : $\delta 1.37$ (3H, s, H-9), 1.34 (3H, s, H-8), 1.60 (m, H-4'), 1.65 (3H, s, H-10), 1.67 (4H, m, H-3' and H-5'), 1.80 (2H, m, H-6), 1.81 (4H, m, H-3 and H-5), 1,93 (1H, m, H-4), 2.02 (4H, m, H-2'and H-6'), 4.12 (1H, m, H-1'), 5.36 $(1 \mathrm{H}$, brs, $\mathrm{H}-2) ;{ }^{13} \mathrm{C}$ NMR $\left(75.5 \mathrm{MHz}, \mathrm{CDCl}_{3}\right): \delta 23.3(\mathrm{C}-$ 10), 24.2 (C-5), 24.7 (C-3' and C-5'), 24.9 (C-8), 25.2 (C9), 25.4 (C-4'), 26.6 (C-3), 31.0 (C-6), 32.9 (C-2' and C6'), 42.7 (C-4), 53.9 (C-1'), 58.3 (C-7), 120.0 (C-2), 134.0 (C-1), $179.7(\mathrm{C}=\mathrm{S})$.

$N$-[1-(4R)-(4-isopropyl-1-methylcyclohexenyl)]-N'-[2(pyrrolidyl)] thiourea (11). Yield: 67\%; IR v $v_{\max } / \mathrm{cm}^{-1}: 3395$ (N-H), 1643 (C=C), 1531 and $1347(\mathrm{C}=\mathrm{S})$; EI-MS m/z (rel. int.): $266\left(\mathrm{M}^{+\bullet}\right) ; 233$ (28); 131 (100, $\mathrm{M}^{+\bullet}$ - 135; [H HCSNH-pyrrolidyl] $^{+}$), 114 (92); 93 (30); 58 (38); 41 (58); ${ }^{1} \mathrm{H}$ NMR $\left(300 \mathrm{MHz}, \mathrm{CDCl}_{3}\right): \delta 1.25$ and $1.78(1 \mathrm{H}$ each, m, H-5), 1.49 (3H, s, H-8), 1.55 (3H, s, H-9), 1.64 (3H, s, H-10), 1.77 and 2.04 (1H each, m, H-3), 1.98 (2H, m, H-6), 1.98 (4H, m, H-2' and H-3'), 2.70 (1H, tdd, $J$ 12.0, 2.4 and 2.1, H-4), 3.55 (4H, m, H-1' and $\mathrm{H}-$ 4'), 5.37 (1H, brs, $\mathrm{H}-2)$; ${ }^{13} \mathrm{C} \mathrm{NMR}\left(75.5 \mathrm{MHz} \mathrm{CDCl}_{3}\right)$ : $\delta 23.3$ (C-10), 24.1 (C-5), 24.6 (C-8), 24.9 (C-9), 25.5 (C-2' and C-3'), 26.6 (C-3), 31.1 (C-6), 40.8 (C-4), 49.2 (4H, m, C-1' and C-4'), 58.9 (C-7), 120.6 (C-2), 134.0 (C-1), $177.3(\mathrm{C}=\mathrm{S})$.

$N$-[1-(4R)-(4-isopropyl-1-methylcyclohexenyl)]-N'-[2-(Nmethylpiperazyl)]thiourea (12). Yield: 90\%; IR $\mathrm{v}_{\max } / \mathrm{cm}^{-1}$ : $3438(\mathrm{~N}-\mathrm{H}), 1629(\mathrm{C}=\mathrm{C}), 1566$ and $1410(\mathrm{C}=\mathrm{S})$; EI-MS $\mathrm{m} / \mathrm{z}$ (rel. int.): $295\left(\mathrm{M}^{+*}\right) ; 195$ (20), 121 (34), 93 (64), 58 (100), 41 (97); ${ }^{1} \mathrm{H}$ NMR (300 MHz, $\left.\mathrm{CDCl}_{3}\right): \delta 1.46(3 \mathrm{H}$, s, H-8), 1.53 (3H, s, H-9), 1.63 (3H, s, H-10), 1.73 (2H, $\mathrm{m}, \mathrm{H}-5), 1.81$ and 1.96 (1H each, m, H-3), 1.96 (2H , m, H-6), 2.30 (3H, s, NC$\left.\underline{H}_{3}\right), 2.43$ (4H, t, J 5.1 Hz, H-2' and H-3'), 2.81 (1H, m, H-4), 3.78 (4H, t, J 5.1 Hz, H-1'and $\mathrm{H}-4$ '), 5.36 (1H, brs, H-2); ${ }^{13} \mathrm{C} \mathrm{NMR}\left(75.5 \mathrm{MHz} \mathrm{CDCl}_{3}\right)$ : б 23.0 (C-10), 23.8 (C-5), 24.2 (C-9), 24.3 (C-8), 26.2 (C-3), 30.7 (C-6), 39.9 (C-4), $45.4\left(\mathrm{NCH}_{3}\right), 46.8$ (C-1' and C-4'), 54.2 (C-2' and C-3'), 58.9 (C-7), 120.3 (C-2), 133.5 (C-1), $180.8(\mathrm{C}=\mathrm{S})$.

N-[1-(4R)-(4-isopropyl-1-methylcyclohexenyl)]-N'-[2(morpholyl)]thiourea (13). Yield: 88\%; IR $v_{\max } / \mathrm{cm}^{-1}: 3408$ (N-H), 1523 and $1347(\mathrm{C}=\mathrm{S}) ;{ }^{1} \mathrm{H} \mathrm{NMR}\left(300 \mathrm{MHz}, \mathrm{CDCl}_{3}\right)$ : $\delta 1.48$ (3H, s, H-8), 1.54 (3H, s, H-9), 1.65 (2H, m, H-5), 1.73 (3H, s, H-10), 2.02 (4H, m, H-3 and H-6), 2.75 (1H, m, H-4), 3.73 (8H, m, H-1', H-2', H-3' and H-4'), 5.36 $(1 \mathrm{H}$, brs, $\mathrm{H}-2) ;{ }^{13} \mathrm{C}$ NMR $\left(75.5 \mathrm{MHz}, \mathrm{CDCl}_{3}\right): \delta 23.3(\mathrm{C}-$ 10), 24.3 (C-5), 24.6 (C-8), 24.7 (C-9), 26.5 (C-3), 30.7 (C-6), 44.5 (C-4), 47.4 (C-1'and C-4'), 59.5 (C-7), 66.3 (C-2'and C-3'), 119.7 (C-2), 134.1 (C-1), $181.8(\mathrm{C}=\mathrm{S})$.

\section{General procedure for S-methylthioureas 14-18}

To a solution of thiourea $(1 \mathrm{mmol})$ in $\mathrm{CHCl}_{3}(10 \mathrm{~mL})$ was added methyl iodide $(5 \mathrm{mmol})$ at $0{ }^{\circ} \mathrm{C}$. The mixture was kept at $0{ }^{\circ} \mathrm{C}$ for $24 \mathrm{~h}$ and then the solvent and excess of methyl iodide were removed under reduced pressure to give the salt of the corresponding S-methylthiourea in quantitative yield for all compounds.

N-[1-(4R)-(4-isopropyl-1-methylcyclohexenyl)]-N'-[2(isopropyl)]-S-methylthiourea (14). IR $v_{\max } / \mathrm{cm}^{-1}: 3193(\mathrm{~N}-$ H), $1600(\mathrm{C}=\mathrm{N})$; EI-MS m/z (rel. int.): $268\left(7, \mathrm{M}^{+*}\right), 220$ (68, $\left.\mathrm{M}^{+\bullet}-\mathrm{CH}_{3} \mathrm{SH}\right), 205$ (57), 128 (100), 127 (60), 93 (77), 58 (91), 43 (96), 41 (85); ${ }^{1} \mathrm{H}$ NMR (300 MHz, $\left.\mathrm{CDCl}_{3}\right): \delta 1.49\left(6 \mathrm{H}, \mathrm{d}, J 6.3 \mathrm{~Hz}, \mathrm{NCH}\left(\mathrm{CH}_{3}\right)_{2}\right), 1.50(3 \mathrm{H}$, s, H-8), 1.58 (3H, s, H-9), 1.65 (3H, s, H-10), $1.76(2 \mathrm{H}$, m, H-5), 1.93 (4H, m, H-3 and H-6), 2.27 (1H, m, H-4), $2.85\left(3 \mathrm{H}, \mathrm{s}, \mathrm{SCH}_{3}\right), 4.30$ (1H, m, H-1'), 5.35 (1H, brs, H- 
2); ${ }^{13} \mathrm{C}$ NMR (75.5 MHz, $\left.\mathrm{CDCl}_{3}\right): \delta 18.4\left(\mathrm{SCH}_{3}\right), 22.7$ $\left(\mathrm{NCH}\left(\mathrm{C}_{3}\right)_{2}\right), 23.3$ (C-10), 24.4 (C-5), 25.1 (C-8), 25.7 (C-9), 26.7 (C-3), 30.8 (C-6), 42.7 (C-4), 51.2 (C-1'), 57.4 (C-7), $120.1(\mathrm{C}-2), 134.1(\mathrm{C}-1), 143.7(\mathrm{C}=\mathrm{N})$.

$N$-[1-(4R)-(4-isoproyl-1-methylcyclohexenyl)]-N'-[2(isopentyl)]-S-methylthiourea (15). IR $v_{\max } / \mathrm{cm}^{-1}: 3347(\mathrm{~N}-$ H), 1690 (C=N); EI-MS m/z (rel. int.): 248 (40, $\mathrm{M}^{+*}$ $-\mathrm{CH}_{3} \mathrm{SH}$ ), 233 (50), 219 (55), 128 (81), 127 (49), 93 (61), 58 (74); 43 (100), 41 (86); ${ }^{1} \mathrm{H}$ NMR (300 MHz, $\mathrm{CDCl}_{3}$ ): $\delta 0.99\left(6 \mathrm{H}, \mathrm{t}, J 7.3 \mathrm{~Hz}, \mathrm{NCH}\left(\mathrm{CH}_{2} \mathrm{CH}_{3}\right)_{2}\right), 1.33(1 \mathrm{H}, \mathrm{m}, \mathrm{H}-$ 5), $1.55(3 \mathrm{H}, \mathrm{s}, \mathrm{H}-8), 1.58(3 \mathrm{H}, \mathrm{s}, \mathrm{H}-9), 1.65(3 \mathrm{H}, \mathrm{s}, \mathrm{H}-$ 10), 1.77 (1H, m, H-5'), 1.79 (4H, m, $\left.\mathrm{NCH}\left(\mathrm{CH}_{2} \mathrm{CH}_{3}\right)_{2}\right)$, $1.94(2 \mathrm{H}, \mathrm{m}, \mathrm{H}-3), 2.01(2 \mathrm{H}, \mathrm{m}, \mathrm{H}-6), 2.26(1 \mathrm{H}, \mathrm{m}, \mathrm{H}-4)$, $2.78\left(3 \mathrm{H}, \mathrm{s}, \mathrm{SCH}_{3}\right), 3.78(1 \mathrm{H}, \mathrm{m}, \mathrm{H}-1$ '), 5.37 (1H, brs, H$2) ;{ }^{13} \mathrm{CNMR}\left(75.5 \mathrm{MHz}, \mathrm{CDCl}_{3}\right): \delta 10.9\left(\mathrm{NCH}\left(\mathrm{CH}_{2} \mathrm{CH}_{3}\right)_{2}\right)$, $18.4\left(\mathrm{SCH}_{3}\right), 23.3$ (C-10), 24.4 (C-5), 25.1 (C-8), 25.6 (C-9), 26.7 (C-3), $27.5\left(\mathrm{NCH}\left(\mathrm{CH}_{2} \mathrm{CH}_{3}\right)_{2}\right), 30.8$ (C-6), 42.8 (C-4), 61.5 (C-7), 63.4 (C-1'), 119.4 (C-2),134.4 (C-1), $169.2(\mathrm{C}=\mathrm{N})$.

$N$-[1-(4R)-(4-isopropyl-1-methylcyclohexenyl)]-N'-[2(cyclohexyl)]-S-methyl-thiourea (16). IR $v_{\max } / \mathrm{cm}^{-1}: 3186$ $(\mathrm{N}-\mathrm{H}), 1685(\mathrm{C}=\mathrm{N}), 1591(\mathrm{C}=\mathrm{C})$; EI-MS $\mathrm{m} / z$ (rel. int.): $308\left(\mathrm{M}^{+*}\right), 261\left(31, \mathrm{M}^{+\bullet}-\mathrm{CH}_{3} \mathrm{~S}\right), 128$ (63), 127 (39), 93 (53), 58 (55), 55 (54), 43 (57), 41 (100); ${ }^{1} \mathrm{H}$ NMR (300 $\left.\mathrm{MHz}, \mathrm{CDCl}_{3}\right): \delta 1.34$ and 1.81 (1H each, $\left.\mathrm{m}, \mathrm{H}-6\right), 1.55$ (3H, s, H-8), 1.57 (3H, s, H-9), 1.62 (2H, m, H-4') 1.65 $(3 \mathrm{H}, \mathrm{s}, \mathrm{H}-10), 1.81(2 \mathrm{H}, \mathrm{m}, \mathrm{H}-5), 1.81(4 \mathrm{H}, \mathrm{m}, \mathrm{H}-3$ ' and H-5'), 1.93 (4H, m, H-2' and H-6'), 1.93 (2H, m, H-3), $2.25(1 \mathrm{H}, \mathrm{m}, \mathrm{H}-4), 2.83\left(3 \mathrm{H}, \mathrm{s}, \mathrm{SC} \underline{\mathrm{H}}_{3}\right), 3.88(1 \mathrm{H}, \mathrm{m}, \mathrm{H}-$ 1'), 5.37 (1H, brs, $\mathrm{H}-2) ;{ }^{13} \mathrm{CNMR}\left(75.5 \mathrm{MHz}, \mathrm{CDCl}_{3}\right)$ : $\delta$ $18.5\left(\mathrm{SCH}_{3}\right), 23.3$ (C-10), 24.4 (C-4'), 24.6 (C-5), 24.8 (C-3' and C-5'), 25.6 (C-8 and C-9), 26.7 (C-3), 30.7 (C-6), 32.6 (C-2' and C-6'), 42.7 (C-4), 56.8 (C-1'), 63.4 (C-7), $119.4(\mathrm{C}-2), 134.3(\mathrm{C}-1), 168.1(\mathrm{C}=\mathrm{N})$.

$N$-[1-(4R)-(4-isopropyl-1-methylcyclohexenyl)]-N'-[2(pyrrolidyl)]-S-methyl-thiourea (17). IR $v_{\max } / \mathrm{cm}^{-1}: 1677$ $(\mathrm{C}=\mathrm{N}), 1584$ (C=C); EI-MS m/z (rel. int.): $280\left(\mathrm{M}^{+*}\right), 142$ (52), 128 (93), 127 (69), 84 (44), 70 (42), 57 (42), 43 (100), 42 (87), 41 (40); ${ }^{1} \mathrm{H}$ NMR (300 MHz, $\mathrm{CDCl}_{3}$ ): $\delta 1.36$ and 1.82 (1H each, $\mathrm{m}, \mathrm{H}-5), 1.60(3 \mathrm{H}, \mathrm{H}-9), 1.62$ (3H, s, H-8), 1.65 (3H, s, H-10), 1.82 (m, H-5), 1.96 (2H, m, H-3), 2.01 (2H, m, H-6), 2.18 (4H, m, H-2' and H-3'), $2.35(1 \mathrm{H}, \mathrm{m}, \mathrm{H}-4), 2.70\left(3 \mathrm{H}, \mathrm{s}, \mathrm{SC}_{3}\right), 4.04(4 \mathrm{H}, \mathrm{m}, \mathrm{H}-$ 1'and $\mathrm{H}-4$ '), 5.37 (1H, brs, H-2); ${ }^{13} \mathrm{C}$ NMR $(75.5 \mathrm{MHz}$, $\left.\mathrm{CDCl}_{3}\right): \delta 17.6\left(\mathrm{~S}_{-} \mathrm{H}_{3}\right), 23.5(\mathrm{C}-10), 24.6(\mathrm{C}-5), 25.4(\mathrm{C}-$ 2' and C-3'), 26.2 (C-8), 26.6 (C-9), 27.1 (C-3), 31.1 (C6), 43.3 (C-4), 53.5 (C-1' and C-4'), 64.1 (C-7), 119.9 (C2), $134.4(\mathrm{C}-1), 164.2(\mathrm{C}=\mathrm{N})$.
$\mathrm{N}-[1-(4 R)-(4-i s o p r o y l-1-m e t h y l c y c l o h e x e n y l)]-N^{\prime}-[2-(N-$ methypiperazyl)]-S-methyl-thiourea (18). IR $\mathrm{v}_{\max } / \mathrm{cm}^{-1}$ : $1675(\mathrm{C}=\mathrm{N}), 1610(\mathrm{C}=\mathrm{C})$; EI-MS m/z (rel. int.): $304\left(\mathrm{M}^{+*}\right)$, 195 (43), 136 (55), 121 (28), 93 (100), 81 (53), 58(16), 44 (56), 41 (75); ${ }^{1} \mathrm{H}$ NMR (300 MHz, $\left.\mathrm{CDCl}_{3}\right): \delta 1.27(3 \mathrm{H}, \mathrm{s}$, H-8), 1.28 (3H, s, H-9), 1.65 (3H, s, H-10), 1.76 (2H, m, H-5), 1.80 and 1.97 (1H each, m, H-3), 1.97 (3H, m, H-4 and $\mathrm{H}-6), 2.34\left(3 \mathrm{H}, \mathrm{s}, \mathrm{NC}_{3}\right), 2.73\left(3 \mathrm{H}, \mathrm{s}, \mathrm{SC}_{3}\right), 3.59$ (4H, m, H-1' and H-4'), 3.70 (4H, m, H-2' and H-3'), 5.37 $(1 \mathrm{H}$, brs, $\mathrm{H}-2) ;{ }^{13} \mathrm{C}$ NMR $\left(75.5 \mathrm{MHz}, \mathrm{CDCl}_{3}\right): \delta 16.9$ $\left(\mathrm{SCH}_{3}\right), 23.4$ (C-10), 24.3 (C-5), 24.6 (C-8), 24.8 (C-9), 26.9 (C-3), 31.3 (C-6), 42.9 (C-1'and C-4'), 46.2 (C-4), 58.8 (C-7), 61.5 (C-2' and C-3'), $52.2\left(\mathrm{NCH}_{3}\right), 121.1$ (C2), $133.9(\mathrm{C}-1), 151.1(\mathrm{C}=\mathrm{N})$.

\section{Antiproliferative assays}

Synthesized compounds were evaluated in vitro against a nine-cell panel lines consisting of melanoma UACC-62, breast MCF7, lung NCI-460, leukemia K562, ovarian OVCAR, prostate PCO-3, colon HT29, renal 786-0 and breast resistant NCI/ADR according NCI standard protocol. ${ }^{17}$ Doxorubicin was used as positive control. Assays were performed in a 96-well plate using four concentrations at 10 -fold dilutions ( $0.25 \mathrm{mg} \mathrm{mL}^{-1}$ to $250 \mathrm{mg} \mathrm{mL}^{-1}$ ) for each test compound. The anticancer activity was deduced from doseresponse curves and three dose response parameters $\left(\mathrm{GI}_{50}\right.$, TGI and $\left.\mathrm{LC}_{50}\right)$ were calculated.

\section{Acknowledgments}

We are grateful to CAPES (Brazil) for the fellowship to I. M. Figueiredo.

\section{References}

1. Carnesecchi, S.; Schneider, Y.; Ceraline, J.; Duranton, B.; Gosse, F.; Seiler, N.; Raul, F.; J. Pharmacol. Exp. Ther. 2001, 298, 197.

2. Bardon, B.; Foussard, V.; Fournel, S.; Loubat, A.; Cancer Lett. 2002, 8, 187.

3. Xu, M.; Floyd, H. S.; Greth, S. M.; Chang, W-C. L.; Lohman, K.; Stoyanova, R.; Kucera, G. L.; Kute, T. E.; Willingham, M. C.; Miller, M. S.; Toxicol. Appl. Pharmacol. 2004, 195, 232.

4. Elegbede, J. A.; Elson, C. E.; Qureshi, A.; Tanner, M. A.; Gould, M. N.; Carcinogenesis 1984, 5, 661.

5. Wattenberg, L.W.; Coccia, J. B.; Carcinogenesis 1991, 12, 115.

6. Crowell, P. L.; Gould, M. N.; Crit. Rev. Oncog. 1994, 5, 1.

7. Kawamori, T.; Tanaka, T.; Hirose, Y.; Ohnishi, M.; Mori, H.; Carcinogenesis 1996, 17, 369. 
8. Chander, S. K.; Lansdown, A.G.; Luqmani, Y. A.; Gomm J. J.; Coope, R. C.; Gould, M. N.; Coombes, R. C.; British J. Cancer 1994, 69, 879.

9. Haag, J. D.; Lindstrom, M. J.; Gould, M. N.; Cancer Res. 1992, $52,4021$.

10. Vigushin, D. M.; Poon, G. K.; Boddy, A.; English, J.; Halbert, G. W.; Pagonis, C.; Jarman, M.; Coombes, R. C.; Cancer Chemother. Pharmacol. 1998, 42, 111.

11. Silva, C. C.; Marsaioli, A. J.; Tetrahedron Lett. 1993, 34, 6717.
12. Oliveira, C. M.; Silva, C. C.; Collins, C. H.; Marsaioli, A. J.; J. Braz. Chem. Soc. 2001, 12, 661.

13. Monks, A. D; Skehan, P; Shoemaker, R; Paull, K; Vistica, D; Hose, C; Langley, J; Cronise, P; Vaigro-Wolff, A; GrayGoodrich, M; Campbell, H; Mayo, J; Boyd, M.; J. Nat. Cancer Inst. 1991, 83, 757 .

Received: January 9, 2006

Published on the web: July 6, 2006

FAPESP helped in meeting the publication costs of this article. 